\title{
Abnormalities in the duodenal transit and motility in duodenal ulcer patients: studies with a new isotopic technique
}

\author{
M G QUON, I MENA, AND J E VALENZUELA
}

From the Gastroenterology Section, Department of Medicine, University of Southern California - Los Angeles County Medical Center, and the Division of Nuclear Medicine, Department of Radiology, Harbor UCLAMedical Center Los Angeles, California, USA.

SUmmary Abnormalities of duodenal motility have been described in patients with duodenal ulcer and in experimental ulcers in rats and it has been postulated that they could be pathogenic in peptic ulcer disease. We have investigated with an isotopic technique whether duodenal bulb clearance or duodenal transit are abnormal in duodenal ulcer. Six patients with inactive and six with active duodenal ulcers, all men, and six healthy male controls were studied. Motility of the duodenum was simultaneously monitored. A bolus of $9 \mathrm{~m}$ TcDTPA was injected into the duodenum while water or acid were perfused on different occasions. Duodenal bulb clearance and transit to the ligament of Treitz were calculated. Duodenal transit in duodenal ulcer patients $108 \cdot 8$ (23) sec was faster than in controls, $194.9(5 \cdot 1)$ sec $(p<0 \cdot 05)$ during the quiescent period of the motility cycle. The frequency of duodenal bulb contractions during acid perfusion was higher in duodenal ulcer patients $1.7(0.4) \mathrm{cont} / \mathrm{min}$, than in controls $0.8(0 \cdot 1)$ cont/min $(\mathbf{p}<0.05)$. No other significant differences were observed between ulcer patients and controls. These data suggest that patients with duodenal ulcers do not have major abnormalities of duodenal bulb clearance, nor of duodenal transit and that duodenal motility does not play a primary role in the pathogenesis of the ulcer.

In spite of considerable progress in the knowledge of the pathophysiology of peptic ulcer disease (PUD), a full understanding of the mechanisms that cause duodenal ulcer (DU) still remains elusive. The most widely accepted concept is that ulcerogenesis involves an imbalance between aggressive factors - for example, acid and pepsin, and protective mucosal factors. ${ }^{1}$ Although more recent findings have enriched our understanding of the mucosal defensive factors ${ }^{23}$ many inconsistencies still exist and a search for additional mechanisms seems warranted. Motility abnormalities are known to be present in DU patients and they could be related to accelerated gastric emptying of liquids found in these patients. ${ }^{45}$ There is some evidence, however, that abnormalities

Address for correspondence: Jorge E Valenzuela, MD, Section of Gastroenterology, Dept of Medicine, USC School of Medicine, 2025 Zonal Avenue, Los Angeles, CA 90033, USA.

Accepted for publication 3 October 1988 of duodenal motility can also be found in DU patients. For instance, Monto and colleagues used manometric methods and reported a decrease in the amplitude and duration of the contractions of the proximal duodenum in DU patients. ${ }^{67}$ These observations were confirmed and expanded by Borgstrom and Arborelius. ${ }^{8}$ These authors used combined fluorography and motility recordings and observed that DU patients have less retrograde motor sequence in the duodenum than control subjects. They postulated that this could represent an important pathogenic mechanism as it prevents the more alkaline content of the distal duodenum from neutralising the acidic gastric content that is delivered to the duodenal bulb. ${ }^{8}$

Interest in this problem has been stimulated more recently after studies on rats with DU induced by the alkyl chemicals, propionitrile and cysteamine. ${ }^{910}$ 579 
cause an increase in the myoelectric activity in the duodenum and a decrease in the bile output from a proximal duodenal cannula." Further studies from the same group characterised the motility changes caused by these duodenal ulcerogens and they proposed that these changes may play a role in the pathogenesis of experimentally induced ulcers. ${ }^{12}$

Duodenal motility changes may lead to decreased intraduodenal mixing of secretions and impaired neutralisation of the proximal duodenal content. Additionally, impaired duodenal motility could decrease the acid wash out so that the bulb in DU patients could be exposed to gastric acid secretion for long periods of time.

We reason that if these abnormalities have a pathogenic role, they would be of greater consequence during fasting when gastric acid secretion is not buffered by the meals. We investigated duodenal transit and duodenal bulb clearance using a new isotopic technique in order to answer the following question: (1) Do patients with DU have abnormalities in the clearance of an isotopic bolus from the duodenal bulb? (2) Are there abnormalities in the duodenal transit flow in DU patients?

\section{Methods}

CONTROLS SUBJECTS

Six healthy male subjects (mean age 35 years, range 25-40) with no history of GI illness and taking no medication regularly were included.

\section{DUODENAL ULCER PATIENTS}

Twelve male patients (mean age 41 years, range $17-$ 63 ) with duodenal ulcer disease were studied. In six, the ulcer was clinically inactive as confirmed by endoscopy and the remaining six were symptomatic and the ulcers active as evidenced by endoscopy. The protocol was approved by the Research Committees of Los Angeles County-University of Southern California Medical Center and Harbor-UCLA Medical Center. Subjects and patients were informed of the nature of the study and they read and signed an informed consent.

DUODENAL MOTILITY AND TRANSIT

As duodenal transit during fasting varies with the different phases of the migrating motility complex (MMC) we identified the phases of the complex before the isotope injection. For this a specially designed double lumen tube was constructed. Two tubes were glued together. One tube consisted of a single polyvinyl catheter (od $0.8 \mathrm{~mm}$ ) with a $1 \mathrm{~mm}$ side port which when placed in the subjects the tips would be located 3-4 cm beyond the duodenal cap and the side port at the level of the duodenal bulb.
This catheter was used for manometric measurements. A second tube consisted of a radioopaque polyurethane tube, od $2 \mathrm{~mm}$ (Norwich-Eaton Pharm, Norwich, NY), with a single $2 \mathrm{~mm}$ side opening at the same level as the polyvinyl tube. This tube was used to inject the radioisotope. A guide wire was passed through the lumen of the polyurethane tube to facilitate positioning in the duodenal bulb. The polyvinyl catheter was perfused with distilled water or an acid solution (see study design below) at a constant rate of $1.6 \mathrm{ml} / \mathrm{min}$ by a Harvard pump (model 2620, Harvard Apparatus, Dover, MA). In line with the polyvinyl tube, an external transducer (Statham P23BD, Hato Rey, Puerto Rico) was attached and duodenal bulb motility recorded on a single channel polygraph (model $7702 \mathrm{~B}$, Hewlett Packard, Walthma, Mass). Sudden occlusion of the side port of the polyvinyl tube caused a pressure rise of about $100 \mathrm{mmHg} / \mathrm{sec}$. After an overnight fasting, the double lumen tube was swallowed and properly positioned in the duodenum as verified by fluoroscopy. Adequate position was verified during the days of study at least twice and whenever this was not considered to be satisfactory, patients were discontinued from the study. This occurred in six additional DU patients. In other instances, if one study was not completed but three studies have been done successfully in a given subject or patient the results were included.

Observation of the manometric tracing in each subject allowed identification of a different degree of peristalsis that presumably represent the phases of the MMC. One period was characterised by virtual absence of peristaltic activity and possibly corresponds to phase I. Another period was identified by irregular contractions of $12 \mathrm{mmHg}$ or more and with a frequency of less than 11 contractions/min. This probably represents phase II and was frequently followed by a burst of contractions with a frequency of about 11 contractions/min, equivalent to phase III (Fig. 1). Because of the short duration of the phase III, we gave intraduodenal infusions of isotope (vide infra) either in the quiet period (phase I) or during the period of irregular activity (phase II).

STUDY DESIGN

The study was done on two different days: on day 1 , after the tube was correctly placed, water at room temperature was perfused through the polyvinyl catheter (basal study). Then $1 \mathrm{mCi}{ }^{*_{m}} \mathrm{TcDTPA}$ bolus, containing approximately $0.03 \mathrm{ml}$ of the isotope dissolved in $10 \mathrm{ml}$ distilled water was injected through the polyurethane tube in about 10 sec either during quiescent or irregular contractions period. One injection was done in each phase on the same day, the sequence of which was randomly selected. 


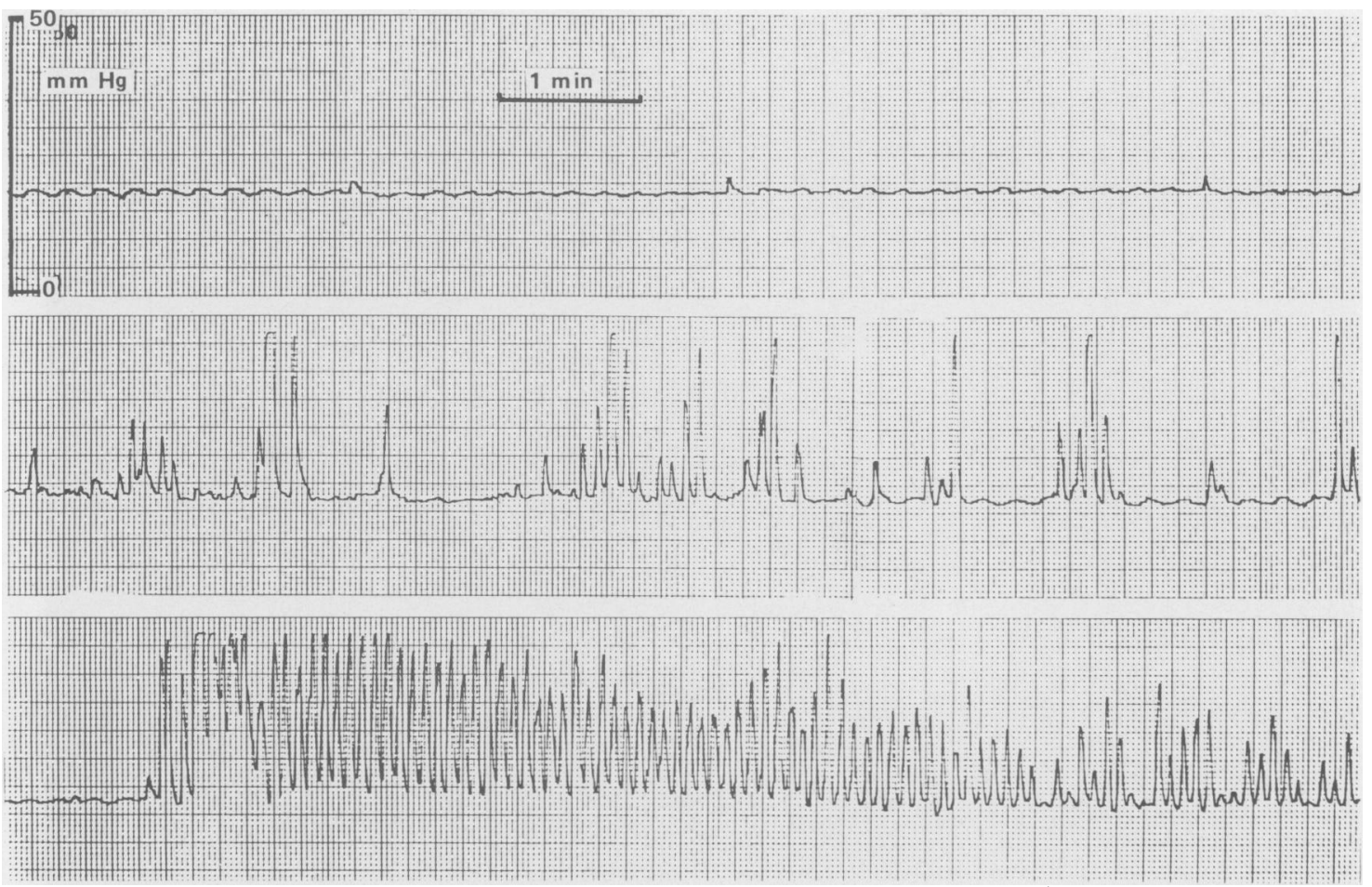

Fig. 1 Representative tracing of the duodenal bulb motility during the quiescent period (upper tracing), period of irregular contraction (middle tracing) and during equivalent to the migrating phase III (lower tracing).

These injections did not cause measurable motility changes. Three hours were allowed between injections to avoid background contamination. On day two, the polyvinyl tube was infused constantly with $52 \mathrm{mM} \mathrm{HCl}$ solution $(\mathrm{pH} \mathrm{1.6)}$ for five minutes. This represents approximately the load of acid delivered to the duodenum in DU patients during fasting, based on acid secretion studies in our laboratory (unpublished data). This infusion was done during each period, preceding the injection of the ${ }^{{ }^{*} \mathrm{~m}}$ TcDTPA bolus which was also done once in each period. The sequence of study days was also selected at random.

DATA ACQUISITON AND ANALYSIS

For each subject, radioactivity over the duodenum was monitored with a gamma camera with a low energy, paralleled hole all purpose collimator. Data were computer acquired in frame mode, and time activity curves were formulated for subsequent analysis. A region of interest was drawn over the duodenum. The level of the ligament of Treitz was estimated 3-6 cm to the left of the second or third lumbar vertebrae. The following parameters were determined: (1) Duodenal transit time (DTT), defined as the time $(\mathrm{sec})$ of initial peak arrival of the isotope at the ligament of Treitz. (2) Duodenal bulb clearance $\left(t^{1} / 2 B C\right)$, time $(\mathrm{sec})$ for one half the isotope to transit away from the duodenal bulb.

Figure 2 shows a composite image after the isotope was injected into the duodenal bulb. This is a typical example of duodenal transit in the quiescent period while water or acid were perfused. Regions of interest are circled around the duodenal bulb and estimated area of the ligament of Treitz so that a time activity curve can be constructed (Fig. 3). During the quiescent period, the isotope typically remained at the region of the duodenal bulb or minimally moved to the more distal portions of the duodenum, but never crossed the midline during the period of observation $(400 \mathrm{sec})$.

During the period of irregular contractions while either water or $\mathrm{HCl}$ was perfused, the isotope after injection moved readily to the distal duodenum and a time activity curve was calculated (Fig. 4). From the data acquired, DTT and $t 12 \mathrm{BC}$ were measured in each subject or patient.

Also a small amount of isotope refluxed to the stomach and this was quantified as duodenogastric reflux and expressed as a percentage of the isotopic activity over the duodenal bulb.

Duodenal bulb contractions higher than $12 \mathrm{mmHg}$ 


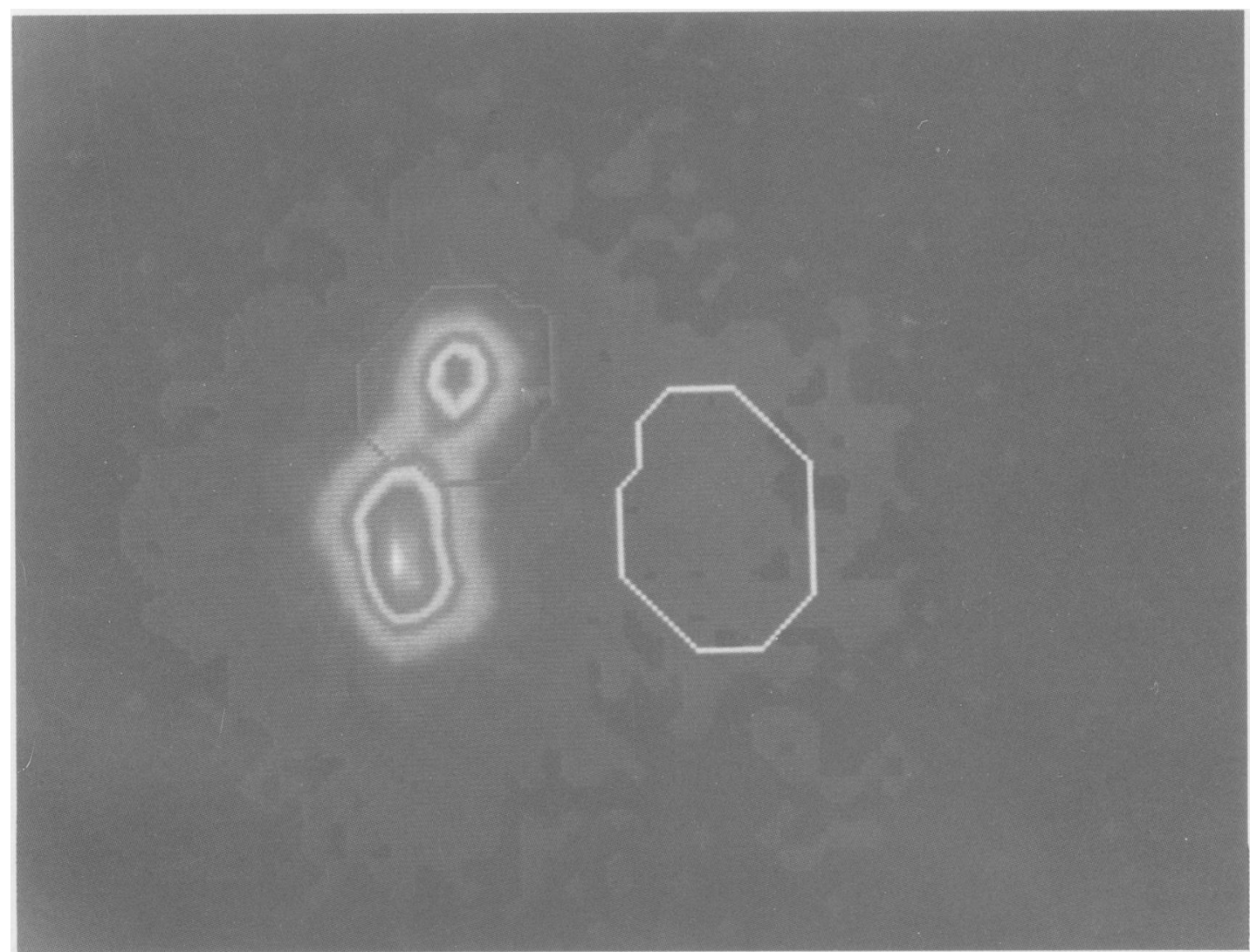

Fig. 2 Photograph of the gamma camera image after injection of the isotope into the duodenal bulb during the quiescent period (phase I of the $M M C$ ). Region of interest at the ligament of Treitz circled in white.

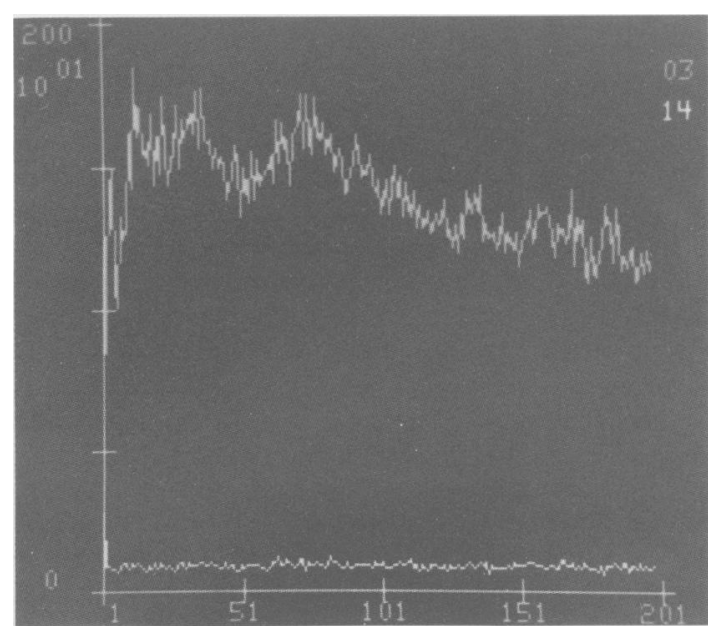

Fig. 3 Time activity curves in one subject at the duodenal bulb (upper tracing) and ligament of Treitz (lower tracing) after the isotope was injected during the quiescent period (phase I). Total period of observation 400 sec:

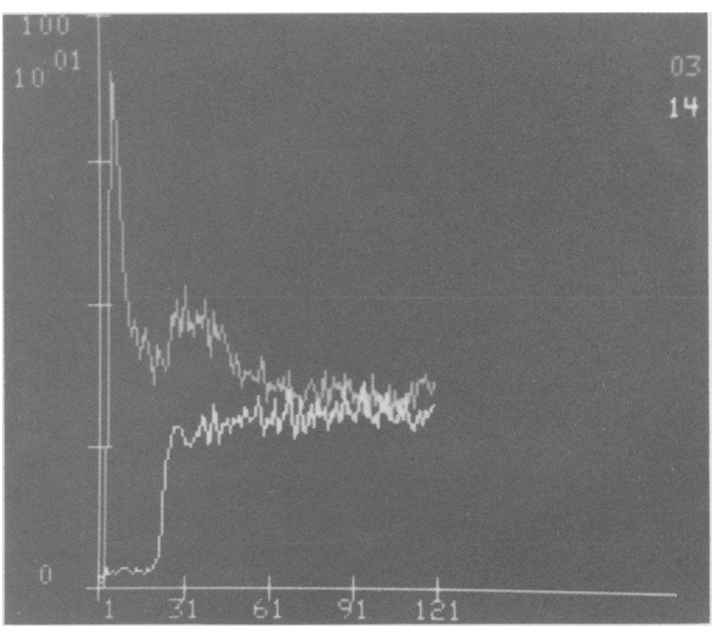

Fig. 4 Time activity curves in one subject after isotope was injected at the duodenal bulb (upper tracing) during the period of contractions (phase II of the MM('). Activity rapidly decreased at the bulb and increased at the ligament of Treitz (lower tracing). Total period of observation toon sece. 
were identified on each tracing. The number of contractions per min calculated and their amplitude averaged to give a single value per subject or patient per study. Means (SEM) were then calculated.

ANALYSIS OF DATA

Duodenal transit time and $\mathrm{t}^{1} / 2 \mathrm{BC}$ expressed in seconds obtained during water and $\mathrm{HCl}$ intraduodenal perfusion and different periods were pooled in the two groups. Means and SEM were calculated. The means were analysed using a non-parametric statistical method, the Wilcoxon's rank-sum test. All values of $\mathrm{p} \leq 0.05$ were considered statistical significance.

\section{Results}

During the period of quiescent activity and water perfusion, modest reflux of the isotope occurred during injection into the bulb (less than $10 \%$ of the total radioactivity over the bulb), in four of the six control subjects. When the injection was done in the same group of subjects during the period of contractions, only one subject had some isotope reflux to the stomach. The same subjects, during perfusion of acid showed no reflux of isotope to the antrum during the period of irregular contractions. A similar pattern with modest reflux during the quiet period and minimal during the irregular period was observed in the DU patients. Two patients with active DU experienced moderate epigastric pain during the acid infusion.

As expected, DTT was significantly shorter during the period of irregular contractions than during the quiet period in both control subjects $(p<0.02)$ and DU patients $(\mathrm{p}<0.001)($ Table $(\mathrm{A}))$. There were no significant differences within each group when DTT

Table Mean duodenal transit time (SE) in control subjects and duodenal ulcer patients during the quiescent period (phase I) and period of irregular contractions (phase II) in the duodenum. Results were obtained during water and 52 $\mathrm{mM} \mathrm{HCl}$ solution infusions $(1.6 \mathrm{ml} / \mathrm{min}$ ) (panel $A$ ). Mean duodenal bulb clearance ( $S E$ ) in both groups during the same experimental conditions (panel $B$ ). ${ }^{*}=p<0.02$ when $D U$ patients and controls were compared.

(A) Duodenal transit time (DTT) (secs)

\begin{tabular}{lcc} 
& Control $(n=6)$ & $D U(n=12)$ \\
\hline Basal: quiescent period & $194 \cdot 9(5 \cdot 1)$ & $108 \cdot 8(23 \cdot 0)^{*}$ \\
Basal: irregular period & $29 \cdot 9(14 \cdot 4)$ & $15 \cdot 1(3 \cdot 5)$ \\
HCL: quiescent period & $149 \cdot 6(32 \cdot 6)$ & $155 \cdot 1(20 \cdot 2)$ \\
HCL: irregular period & $12 \cdot 3(3 \cdot 0)$ & $18 \cdot 6(4 \cdot 4)$
\end{tabular}

(B) Duodenal bulb clearance $\left(t^{1 / 2 B C}\right)($ secs)

Basal: quiescent period $\quad 121.0(21.7)$

Basal: irregular period $\quad 13.5(4.3)$

HCL: quiescent period $\quad 78.1(27.9)$

HCL: irregular period $\quad 9.0(2 \cdot 1)$

$65 \cdot 3(15 \cdot 2)$

$22 \cdot 5(9 \cdot 1)$

$73 \cdot 8(18 \cdot 2)$

$8 \cdot 4(3 \cdot 3)$ was compared during water and acid perfusion. When controls and DU patients were compared, however, only DTT during the basal quiescent phase was significantly shorter in DU patients $(p<0.02)$. Similarly, $t \frac{1}{2} B C$ was significantly shorter during the period of irregular contractions than during the quiet period in both controls $(p<0.05)$ and DU patients $(p<0.05)$ (Table $(B))$. There were no significant differences within each group, however, when $t^{1} / 2 B C$ was compared during water and acid perfusion. All other values for DTT and $t^{1} / 2 B C$ between controls and DU patients and between water and acid perfusion were not significantly different (Tables (A) and (B)). Also when values observed in active DU patients were compared with those with inactive ulcers, no statistically significant differences were found (results not shown).

No significant differences were found when the amplitude of duodenal bulb contractions during the irregular period were compared during water perfusion in controls $23.3(2 \cdot 8) \mathrm{mmHg}$ and acid perfusion, $28.8(3.9) \mathrm{mmHg}$; with those observed in DU patients during water perfusion $27.6(2.2 \mathrm{mmHg}$ $(p>0.1)$ and acid perfusion $24.7(1.5) \mathrm{mmHg}$ $(p>0.1)$, respectively. Also the frequency of duodenal bulb contractions during water perfusion in controls $0.9(0.2)$ cont $/ \mathrm{min}$ were not significantly different from that of DU patients under similar water perfusion, $1.2(0.2)$ cont $/ \mathrm{min}$. By contrast, the frequency of duodenal bulb contractions during acid perfusion in DU patients $1.7(0.4)$ cont $/ \mathrm{min}$ was significantly higher than that of controls, $0 \cdot 8(0 \cdot 1)$ cont $/ \min (p \leq 0.05)$ during the same perfusion.

\section{Discussion}

Attempts to elucidate the pathogenesis of PUD are limited by the difficulty in getting a close insight into the biochemical, functional, and morphological changes that occur during the development of an ulcer crater. It seems, therefore, of interest to examine experimental models of peptic ulcer. Although there may be differences between the lesions in animals and those seen in man, the ulcers in animal models give us the unique opportunity to evaluate events that occur during the development of the ulcer. Szabo et al have developed a model in rats with DUs induced by cysteamine and propionitrile. ${ }^{10}$ They recently observed that both ulcerogens also induce duodenal hypermotility and decrease the amount of bile drained through a proximal duodenal fistula. " These authors proposed that hypermotility could play a predisposing role in experimental ulceration by preventing mixing and neutralisation of the acid with duodenal alkaline secretions. ${ }^{12}$

Is there an equivalent situation in patients with 
DU? Early manometric studies by Monto et al described hypomotility of the proximal duodenum as compared to the distal duodenum in DU patients. ${ }^{7}$ Using a combined manometric and radiological technique, Borgstrom and Arborelius described a lower number of retrograde motility events in DU patients and postulated that these patients have decreased intraduodenal reflux, with impaired mixing of secretions. They postulated this could have pathogenic importance in DU patients. ${ }^{*}$

In this article, we have examined this hypothesis in patients with active and inactive PUD and also the possibility that these patients could have delayed clearance of the duodenal bulb. Except for DTT during water perfusion in the quiescent phase, we were unable to show either a failure of the duodenal bulb clearance mechanism or consistent alterations in duodenal transit. To approach this problem we developed a new manometric and isotopic technique with intubation of the duodenal bulb. This method allows accurate measurement of the contractions and net movement of the isotopic bolus. It does not allow assessment of to-and-fro or mixing movements. An essential prerequisite in this method is the assurance that the manometric recording and injection of the isotope takes place into the duodenal bulb. This was assured by fluoroscopic control before the injection of ${ }^{{ }^{m} \mathrm{~m}}$ TcDTPA. Also, as fasting motility of the duodenum varies with the different phases of the MMC, the motility of the duodenal bulb was monitored simultaneously and equivalent phases of the MMC were identified by manometric recording. Furthermore, to reproduce the conditions as to how the duodenum of DU patients handles gastric emptying during fasting, the duodenal bulb was perfused with an acid solution similar to the gastric content delivered to the duodenum in fasting.

The shorter DTT during the quiescent period in DU patients as compared with control subjects seems to confirm earlier findings that duodenal peristalsis is accelerated in these patients. These data would also support Szabo et al's proposal that DU patients may have inappropriate mixing of duodenal bulb content with pancreatic and biliary bicarbonate that are secreted into the second portion of the duodenum. ${ }^{11} 12$ This difference, however, was not observed during the period of irregular contractions, equivalent to phase II of the cycle. The relevance of this accelerated transit in the regulation of duodenal bulb $\mathrm{pH}$ during fasting appears then of lesser importance. Overall, the quiescent period comprises less than $15 \%$ of the time of the entire cycle among controls and PUD patients studied in our laboratory (unpublished observations). The remainder of the time the duodenal motility corresponds to the irregular contractions period, equivalent to either phase II or the migrating phase III. Furthermore, when acid was infused into the duodenum to mimic emptying of gastric acid content, DTT in DU patients became almost identical to that of controls.

On the other hand, studying duodenal bulb clearance does not reveal any significant differences between DU patients, active or inactive, and controls. This suggests, on one hand, that stasis of gastric acid in the duodenal bulb does not play an important role in the pathogenesis of DU. On the other, the finding of higher frequency of duodenal bulb contractions during acid perfusion in DU patients does not determine $t^{1} / 2 B C$ in these patients.

By studying both active and inactive DU patients we have eliminated the possibility that changes in duodenal motility could only occur while the ulcer crater is present. We could not, however, exclude that transient motility changes could take place during the formation of the ulcer. It is also well established that duodenal ulcer is a multifactorial disease $^{13}$ and it could be postulated that duodenal motility abnormalities could play a pathogenic role only in some patients. Careful analysis of the individual results fails to identify patients that, with the present technique, show a distinct duodenal transit pattern, and could constitute a subgroup.

In summary, using a isotopic technique we have not demonstrated in DU patients a significant difference in the clearance of an isotopic bolus from the duodenal bulb nor in the flow through the distal duodenum as compared with controls. These findings do not support the hypothesis that duodenal motility changes play a pathogenic role in DU.

The authors are grateful to Arnulfo Pleyto and George Franklin for technical help, to Dr Leslie Bernstein for assistance in the statistical analysis, and to Maria L Vidrio for preparing the manuscript.

\section{References}

1 Grossman MI. The pathologic physiology of peptic ulcer. Am J Med 1960; 29: 748-53.

2 Isenberg JI, Selling JA, Hogan DL, Koss MA. Impaired proximal duodenal mucosal biocarbonate secretion in patients with duodenal ulcer. $N$ Engl J Med 1987; 316: 374-9.

3 Quigley EMM, Turnberg LA. pH of the microclimate lining human gastric and duodenal mucosa in vivo studies in control subjects and in duodenal ulcer patients. Gastroenterology 1987; 92: 1876-84.

4 Malagelada JR, Longstreth GF, Deering TB, Summerskill WHJ, Go VLW. Gastric secretion and emptying after ordinary meals in duodenal ulcer. Gastroenterology 1977; 73: 980-94.

5 Lam SK, Isenberg JL, Grossman MI, Lane WH, Hogan DL. Rapid gastric emptying in duodenal ulcer patients. Dig Dis Sci 1982; 27: 598-603. 
6 Monto GL, Ashworth WE, Malecki M. Thompson AB. Englert E. Duodenal contraction wave patterns in patients with duodenal ulcer. Am J Gastroenterol 1976; 65: 52-6.

7 Monto GL, Ashworth WD, Malecki M, Thompson AB, Englert E. Comparative duodenal motility in patients with and without duodenal ulcer disease. Am J Gastroenterol 1974; 61: 113-8.

8 Borgstrom S, Arborelius M. Duodenal motility pattern in duodenal ulcer disease. Scand J Gastroenterol 1978; 13: $349-52$.

9 Szabo S, Selye H. Duodenal ulcers produced by propionitrile in rats. Arch Pathol 1972; 93: 390-1.

10 Szabo S, Reynolds ES, Lichtenberger LM, Haith LR, Jr,
Dzan VJ. Pathogenesis of duodenal ulcer: gastric hyperacidity caused by propionitrile and cysteamine in rats. Res Commun Chem Pathol Pharmacol 1977; 16: 311.

11 Szabo S, Pihan G, Gallagher GT, Brown A. Role of local secretory and motility changes in the pathogenesis of experimental duodenal ulcer. Scand J Gastroenterol 1984; 19 [suppl 92]: 106-11.

12 Pihan G, Kline TJ, Hollenberg, Szabo S. Duodenal ulcerogens cysteamine and proprionitrile induce gastroduodenal motility alterations in the rat. Gastroenterology 1985; 88: 989-97.

13 Grossman MI, Kurata JH, Rotter JL, et al. Peptic ulcer: new therapies, new diseases. Ann Intern Med 1981; 95: 609-27. 